\title{
EDUCACIÓN AMBIENTAL A PARTIR DE TRES ENFOQUES: COMUNITARIO, SISTÉMICO E INTERDISCIPLINARIO
}

\author{
Onelia Covas Álvarez \\ Diplomante en Dirección Científica, ISP "Pepito Tey"; Cuba
}

\section{INTRODUCCIÓN}

El hombre, desde su surgimiento ha disfrutado de las riquezas de a naturaleza, pero en esos momentos no las afectaba en un grado considerable. En las etapas posteriores del desarrollo de la sociedad humana fue creciendo la capacidad del mismo de modificar su medio ambiente, y consecuentemente, se presentó la necesidad de salvaguardar la naturaleza de los efectos nocivos de esta actividad.

Es a inicios de la segunda mitad del siglo XX, cuando se comienzan a percibir problemas de carácter inéditos que afectan a la propia supervivencia de la especie humana sobre la tierra.

Nuestra sociedad está llamada a la formación de ciudadanos capaces de desempeñar la función que le corresponde en favor del planeta y de su entorno socio-natural, basados en sólidos conocimientos, principios y convicciones, garantizando el equilibrio relacional de la pluralidad de factores bióticos, abióticos y sociales constituyentes de nuestro espacio vital.

La educación como proceso y a la escuela como institución, juegan un papel esencial en esta batalla, puesto que deben involucrar a todos los miembros de la sociedad en la búsqueda de soluciones para resolver los problemas del medio ambiente, proporcionándoles el conocimiento, las habilidades y las motivaciones necesarias par una adecuada interpretación del mundo y una actuación social consecuente con sus necesidades y exigencias.

Para garantizar que la educación alcance ese propósito esencial, se requiere de la introducción formativa de la dimensión ambiental en su integralidad socio-cultural. Esta realidad implica un tratamiento de la problemática ambiental de manera coherente y significativa, que propicie que la actividad cognoscitiva de los alumnos se encuentre en constante desarrollo para integrar conocimientos.

Desde su concepción en Estocolmo, la Educación Ambiental ha sido un proceso educativo permanente en aras de lograr que los individuos y la sociedad en general tomen conciencia de su medio y que adquieran conocimientos, habilidades y valores, que le permitan desarrollar un papel positivo, tanto individual como colectivo hacia la protección cel medio ambiente y el mejoramiento de la calidad de vida humana.

Se evidencia entonces, que la Educación Ambiental no presenta barreras de edad, ni de sistema educativo, por lo que, en cualquier momento el individuo es capaz de orientar de forma positiva sus impresiones y valores respecto al medio ambiente.

Esta conceptualización, conduce a un análisis de las posiciones existente en torno a cómo introducir lo ambiental en el Proceso Docente Educativo y la correspondiente asunción de criterios para la 
fundamentación de la propuesta para su introducción; la dimensión ambiental, la concebimos como: un enfoque que en un proceso educativo, de investigación, se expresa por el carácter sistémico de un conjunto de elementos que tienen una orientación ambiental determinada: expresada a través de los vínculos medio ambiente y desarrollo; los que consecuentemente están interconectados, donde las funciones o comportamientos de unos, actúan y pueden modificar las de los otros.

Es decir, que la dimensión ambiental en un proceso educativo posee un carácter sistémico, interdisciplinario y comunitario, que más adelante se llamarán enfoques por la manera en que se trabajará en está investigación.

En el caso de la dimensión ambiental de un plan de estudio, por ejemplo, su introducción consistirá en la incorporación de una concepción integradora de conocimientos, hábitos, habilidades, actitudes y valores, concientemente diseñado y contextualizado, que atraviese todo el plan de estudio, de manera que quede establecido que aportó cada uno de estos contenidos en el proceso docente educativo y que dé como resultado una formación integral en el sujeto, que se exprese en su actuación hacia el medio, respecto a su entorno y a la problemática ambiental.

Por tanto, se deberá tener en cuenta que la introducción de la dimensión ambiental en el sistema educativo, llevará consigo cambios en la teoría y metodología del plan de estudio, que potencien la valoración crítica, la modificación de actitudes, valores y el desarrollo de comportamientos responsables hacia el medio ambiente y su entorno en especial.

La Educación Ambiental, además de ser un proceso de aprendizaje permanente, donde se afirman valores, es un proceso dirigido a mejorar la calidad de vida y las condiciones de la población, las relaciones humanas, su cultura y su entorno, reconocerlo como recurso educativo; proteger al medio ambiente y comprender las relaciones entre el hombre la naturaleza y la sociedad.

A escala nacional e internacional se ha trabajado la Educación Ambiental a partir de el enfoque interdisciplinario, multidisciplinario y trandisciplinario, además del enfoque comunitario, nosotros a través de este artículo pretendemos contribuir a la formación de la misma a través de la integración de los enfoque comunitario, sistémico e interdisciplinario.

El enfoque comunitario un enfoque muy trabajado en estos últimos tiempos, producto a la necesidad de incidir en nuestros educandos con el objetivo de formar actitudes y valores medioambientales para apaciguar la crisis y lograr transformar la actitud depredadora del hombre, por los problemas ecológicos existente en nuestro planeta.

La Educación Ambiental debe desarrollar en los alumnos la capacidad de observación crítica, de comprensión y de responsabilidad hacia el medio ambiente, que se caracteriza por su multivariedad. Un principio fundamental de ésta es el de la contextualización del contenido al medio ambiente donde vive el escolar, de ahí que sea por excelencia comunitaria, pues la comunidad es su campo fundamental y sus problemas deben formar parte del contenido de las actividades.

Los problemas y sus causas deben ser estudiados y analizados desde lo local a lo global con una progresión de continuidad conectada: micro, macro y viceversa. Partir de la solución de los problemas cercanos de la vida del centro o comunidad, es decir, ubicar a os alumnos frente a las realidades ambientales locales y a partir de ellas, se puede ir adentrando en otras ya sea regionales o globales. 
Un error que se comete en el tratamiento de este enfoque, es el de concederle importancia a los fenómenos y problemas ambientales que ocurren a escala internacional, no es que no se tenga en cuenta estos problemas que ocurren en el mundo, pues se hace necesario conocerlos, porque estos inciden sobre nuestro planeta y nos afectan también, sino, que se debe tener en cuenta los problemas que ocurren en nuestro país, nuestra provincia, en el municipio y más específico en el entorno de la escuela, la comunidad donde vivimos, para conocer las causas que los provoca y sus posibles soluciones.

Si se quiere lograr una conciencia de los principales problemas de la comunidad se han de realizar actividades con los alumnos que le permitan identificar dichos problemas, analizar las causas de su surgimiento, las consecuencia para la vida de la comunidad y su implicación en la solución práctica de ellas, cuestión que se tiene muy en cuenta en los objetivos de la educación para todos los niveles de enseñanza del país y en esta investigación.

En la actualidad no se discute si el medio ambiente es mucho más que naturaleza o ecosistema natural, o sea, es un complejo sistema íntimamente relacionado donde se tiene en cuenta diferentes elementos concatenados como el hombre, la naturaleza, la sociedad, las relaciones sociales, culturales, etc.

Es menester que se comprenda la responsabilidad que se debe asumir ante el medio ambiente y del por qué es necesario favorecer la Educación Ambiental de los estudiantes en el contexto institucional y social como espacio más general de actuación, es por ello la necesidad de este enfoque comunitario

El trabajar este enfoque en el Proceso Docente Educativo, implica la integración sistémica y sistemática de la Educación Ambiental, desde una perspectiva de vinculación "Medio Ambiente-EscuelaComunidad", de ahí la importancia de la contextualización del medio donde está enclavada la escuela, de insertar a través del sistema educativo contenidos medio-ambientales para formar en nuestros educandos una cultura medio-ambiental, para lograr conductas correctas hacia el entorno.

Por ello se hace necesario trabajar en la Educación Ambiental el enfoque sistémico.

Todos los problemas medioambientales tienen necesariamente unan constitución sistémica, al considerarlos como un todo organizado, compuesto por partes que interactúan entre sí. Por tanto, entender el Medio Ambiente como un sistema en el que los elementos que lo integran se encuentran interrelacionando, es una característica fundamental de la Dimensión Ambiental. También el Medio Ambiente manifiesta una visión sistémica, donde los componentes de dicho sistema están integrados en el medio físico, biótico, económico y sociocultural.

Como se aprecia, la característica fundamental del enfoque sistémico no está dada tanto en la composición de los elementos que integran sus partes, sino en cómo se integran estas partes entra sí para formar una unidad dialéctica, (de manera tal que el cambio que se produzca en alguno de sus elementos afecta a los demás) y cómo la integración entre ellas conduce al desarrollo.

A partir de este análisis, se puede plantear que un proceso de Enseñanza - Aprendizaje basada con visión sistémica, se debe caracterizar por:

- La integración de los elementos que la constituyen.

- El enriquecimiento recíproco de las materias que se relacionan. 
- Una concepción holística de la realidad.

- La transformación de los estilos de enseñanza y aprendizajes tradicionales, para producir cambios desde el punto de vista didáctico, lo cual necesariamente lleva a formular proyectos, programas y estrategias educativas que respondan a o realidades necesidades.

En diferentes investigaciones se ha abordado lo relacionado con esta terminología o teoría, entendemos al sistema como: "un conjunto de elementos que se relacionan entre ellos y con el medio". La aceptación científica de este planteamiento concede operatividad epistemológica y metodológica al enfoque sistémico, entendido también como: " un paradigma de carácter, en tanto representa una completa constelación de creencias, valores, técnicas y visión del mundo, compartidos por unos miembros de una determinada comunidad".

El enfoque sistémico como concepción científica, evidencia una dualidad instrumental de inobjetable valor: "como metodología de análisis descriptivo y como estrategia de optimización del sistema". Por esa razón, antes de definir el Medio Ambiente, se hace necesario analizar desde el punto de vista filosófico la definición de sistema: "conjunto de elementos relacionados entre sí, que constituyen una determinada formación íntegra". Estas son razones por las cuales el enfoque sistémico sustenta la base teórica del tratamiento a los problemas del Medio Ambiente, el cual ha transitado por diferentes momentos en su evolución histórica.

"El enfoque sistémico aparece como un instrumento metodológico cuyo objeto es identificar en u marco coherente el conjunto de factores, estados e interacciones que caracterizan la aparición de un fenómeno de la existencia de cualquier problema ambiental".

El enfoque sistémico en la Educación Ambiental requiere un proyecto planteado desde una visión global que considere que se trata de un sistema abierto en el que el todo es más que la suma de sus partes, en el es más explicativo el conocimiento de las interrelaciones, donde se busca el tratamiento interdisciplinario, se valora la estructura y funcionamiento, teniendo en cuenta aspectos dinámicos, evolutivos y la realización del sistema dada su complejidad.

El gran desafío de la Educación Ambiental es saber captar la totalidad en movimiento fluyente, lo que supone un modelo de enseñanza-aprendizaje en el que no se proponen conocimientos adicionales y yuxtapuestos, si no que se precisa establecer conexiones y relaciones de los saberes en una totalidad no dividida y en permanente cambio. Este tratamiento integrador de los conocimientos puntuales exigen un enfoque interdisciplinario.

\section{Enfoque interdisciplinario.}

La interdisciplinariedad, representa un conjunto de disciplinas conexas entre sí y con relaciones definidas, a fin de que sus actividades no se produzcan en forma aislada, dispersas y fraccionadas. Ella nace con el carácter individual de diversas asignaturas que ponen en evidencias sus interdependencias y con ellas se logra dar una visión global y menos esquemáticas de los problemas. Es decir la articulación de las diferentes disciplinas a fin de comprender un proceso en su totalidad, para pasar a continuación al análisis y la solución de un problema en particular. 
La incorporación de éste enfoque interdisciplinario a la práctica educativa, se debe realizar gradualmente, lo cual presupone la realización de colectivos pedagógicos, de años y de disciplinas, en aras de lograr una organización adecuada de la enseñanza, que contribuya a que los alumnos y alumnas comprendan la estructura compleja del medio ambiente, tal como resulta de la interacción de sus aspectos físicos, biológicos, sociales y culturales, así como proporcionar una conciencia clara de la interdependencia política, económica y ecológica del mundo.

Por tanto se trata de permitir tomar conciencia de los problemas que suponen obstáculo al bienestar individual y colectivo, investigar sus causas y determinar las vías para resolverlos. Así podrán participar en una definición colectiva de estrategias para resolver los problemas que afectan la calidad del medio ambiente.

Por interdisciplinariedad se entiende como "....metodología que caracteriza a un proceso docente, investigativo o de gestión, en el que se establece una interrelación de coordinación y cooperación efectiva entre disciplinas, pero manteniendo sus marcos teóricos-metodológicos...", concepto al cual se adscribe la investigadora, por su gran connotación desde el punto de vista metodológico para implementar en la práctica pedagógica.

Los procesos de integración interdisciplinaria suponen una relación más orgánica entre las asignaturas, donde cada una de ellas aporta esquemas conceptuales, métodos de integración y formas para analizar los problemas mediante una estrecha y coordinada cooperación.

El principio filosófico dialéctico materialista de concatenación de los fenómenos se refleja en la enseñanza a través de los contenidos comunes a varias asignaturas, en el Proceso Enseñanza Aprendizaje. El progreso el conocimiento se alcanza en el movimiento del pensar, que pasa de nexos menos profundos y generales a establecer nexos más profundos y específicos entre los hechos, procesos, fenómenos de éste mundo infinito.

La interdisciplinariedad no es solo criterio epistemológico, un sistema instrumental y operativo sino también una forma de ser. Ella expresa el carácter múltiple de las relaciones y la orientación del sentido de acuerdo con los ordenes que vaya estableciendo.

La interdisciplinariedad de la Educación Ambiental a través de esta investigación se trabajará a partir de cómo insertar a través del sistema educativo contenidos medioambientales para formar en nuestros educandos una cultura ambiental y lograr conductas correctas hacia el entorno, no es solo conocerlo, es decir, no basta con educar para la naturaleza utilizándola como recurso educativo, sino:

- Se trata de educación sobre el medio: donde se trate cuestiones ambientales en el aula o taller (sobre todo en los entornos natural y urbano).

- La educación en el medio: se realice un estudio del medio en que está enclavada la escuela, del entorno que rodea a los educandos en el que se desenvuelven, todo esto desde el punto de vista naturalista.

- La educación para el medio: desemboca en una acción tendente al cambio de actitudes, a la formación de valores, para conservar el medio natural y/o urbano, para incidir sobre él con un espíritu de conservarlo si no esta dañado o de transformarlo si lo está. 
Partiendo de lo anteriormente planteado puede afirmarse que la escuela como institución educativa, es la encargada de formar una personalidad integral, capaz de propiciar un desarrollo con sustentabilidad, a través un proceso pedagógico planificado, organizado y coherente.

Para ello necesita un maestro con un alto nivel de integridad, que garantice además de los conocimientos necesarios, el desarrollo de habilidades y la formación de valores que hoy exige nuestra sociedad para el cuidado y conservación de nuestro entorno, un maestro que eduque ambientalmente a nuestros estudiantes. De ahí la necesidad de incluir en el currículo la dimensión ambiental con una perspectiva holística, desarrolladora, tal como se establece en los principios de la Educación Ambiental hacia sociedades sustentables.

La Educación Ambiental debe materializarse teniendo en cuenta el sistema de influencias educativas, donde el núcleo lo constituya la escuela, en la que el docente mediante las indicaciones metodológicas propuesta logra el desarrollo del pensamiento crítico y con esto el alumno se sienta responsable ante el medio ambiente del cual forma parte.

El desarrollo de un modelo didáctico interdisciplinario donde sé interrelacione los enfoques (sistémico, comunitarios e interdisciplinario) de la Educación Ambiental posibilita una mayor orientación, interacción profesor -alumno y articulación entre el conocimiento y las actitudes medioambientales.

Los estudiantes, al mantenerse como protagonistas del proceso, identifican los problemas medioambientales relacionado con el contenido objeto de estudio, realizan valoraciones, análisis y llegan a proponer acciones, o sea, que pasan por diferentes estadios donde manifiestan sus acciones transformadoras ante el medio ambiente.

Teniendo en cuenta las consideraciones que deben ser retomadas de la Educación Ambiental para esta investigación, existe la necesidad de lograr en los educandos un aprendizaje que provoque en estos un cambios en el comportamiento y la resignificación de los valores.

La inclusión de lo ambiental en los programas de las asignaturas de ciencias exactas en la enseñanza preuniversitaria, conlleva a cambios significativos en el sistema educativo, desde sus fines hasta los contenidos y metodologías de su enseñanza, de manera que se redefina el tipo de persona que se quiere formar, en función de los futuros escenarios de su desempeño.

\section{BIBLIOGRAFÍA}

Bustos, Hortensia. Programa de Educación Ambiental (Educación para la Ciudadanía). En http://www.Programa de Reforma del achillerato1.uandecua.htm, oct, 2003.

CAstillo, S. / Pedagogía Ambiental. La tarea de los educadores. En http.//www.casapazcl/biblioteca/mosaicos/m38/portada.htm.15nov, 2002.

Educación Ambiental. Principios de la Enseñanza Aprendizaje. Serie20. Ed. Libros de la Cataratas . España. (PIEA-UNESCO-PNUMA, 1993). 190pa.

ESTRATEGIA NACIONAL, La Habana, 1997, p.19

Guía CURRICULAR EN EDUCACIÓN AMBIENTAL PARA MAESTROS DE CIENCIAS DE 7ํ GRADO. Instituto de Educación Ambiental. Universidad Metropolitana. CINEDA Edición Revisada. 1992. p.54. 
I CONVENCIÓN INTERNACIONAL SOBRE MEDIO AMBIENTE Y DESARROLlo. Memorias de Educación Ambiental para el Desarrollo Sostenible a 20 años de Tbilisi. Ministerio de Ciencias Tecnologías y Medio Ambiente. Editorial Academia, La Habana, 1998 p.256.

MiRANDA Vera, C. Filosofía y Medio Ambiente. Una aproximación teórica. México: Ed Taller Abierto, p149. 1997

NúÑEZ COBAS, Nelson: La Educación de actitudes medioambientales en estudiantes de la especialidad de Química Industrial de la Educación Técnica y Profesional. Tesis en opción al grado científico de Doctor en Ciencias Pedagógicas. Instituto Superior Pedagógico "José de la Luz y Caballero", Holguín, 2003

OMEGNA, Gabriela. Evolución de la Educación Ambiental. En http://www Fundación Vida Silvestre Argentina.htm, dic, 2003.

PEDROSA FLORES, René. Interdisciplinariedad y transdisciplinariedad en los modelos de enseñanza de la cuestión ambiental. En http://www.moebio.uchile.cl/15/frames02.htm, 5dic,2002. 


\title{
Contactar
}

Revista lberoamericana de Educación

\author{
Principal OEI
}

